\title{
Earthquake Fragility Assessment of the Underground Tunnel Using an Efficient SSI Analysis Approach
}

\author{
Thai Son Le ${ }^{1}$, Jungwon Huh1 ${ }^{*}$, Jae-Hyun Park ${ }^{2}$ \\ ${ }^{1}$ Department of Civil and Environmental Engineering, Chonnam National University, Yeosu, Korea \\ ${ }^{2}$ Department of Geotechnical Research Engineering, Korea Institute of Civil Engineering and Building \\ Technology, Goyang, Korea \\ Email: ${ }^{* j w o n h u h @ c h o n n a m . a c . k r ~}$
}

Received September 2014

\begin{abstract}
This paper represents a simplified seismic fragility analysis approach of the underground tunnel structure in consideration of the soil-structure interaction (SSI) effect. SSI effect founds to be essential in the estimation of dynamic analysis of underground structures like tunnels and thus needs to be considered. The ground response acceleration method for buried structures (GRAMBS) known to be a very efficient quasi-static method that can consider SSI effect is used in the proposed approach to evaluate seismic structural responses without sacrificing much accuracy. Seismic fragility curves are then developed by applying the maximum likelihood estimates (MLE) to responses of a large set of artificial ground motion time histories generated for multiple different levels of earthquake intensity. It is also assumed in this paper that the seismic fragility curve can be represented by a two-parameter lognormal distribution function with median and log-standard deviation that need to be defined using MLE.
\end{abstract}

\section{Keywords}

Soil-Structure Interaction, Maximum Likelihood, Fragility Curves, Dynamic Analysis

\section{Introduction}

So far, soil-structure interaction (SSI) analysis is one of the main methods which are being used to observe the structural behavior under seismic excitation considering interaction effect between soil and structure. Also it is known that, due to unbounded nature of ground system, the computational size of this method is very large. For this reason, the GRAMBS that can reduce the computational cost of analysis has been proposed [1]. In addition, there is a necessity of a comprehensive methodology for risk assessment of tunnel structure, which has been mainly based on the expert judgment or empirical fragility curves derived from actual damage record in past earthquake. The study presents the development of fragility curves by integrating a simplified SSI analysis me-

${ }^{*}$ Corresponding author. 
thod, RAM and maximum likelihood estimates (MLE).

\section{A Simplified Seismic Fragility Analysis Methodology}

\subsection{Response Acceleration Method-RAM}

A simplified dynamic analysis approach GRAMBS is used for two dimensional seismic SSI analyses of tunnel structures. First, the surrounding soil of the tunnel structure is subdivided into several layers and the free-field soil response analysis without considering the tunnel is performed using the equivalent linear method-based software such as SHAKE, M-SHAKE, etc. The time dependent displacement and acceleration response of each layer are stored. Then, the time $t_{m}$ when the maximum displacement difference occurs between the top and bottom levels of the tunnel is sought. The response acceleration at $t_{m}$ is taken over the full depth of the soil column and converted into the body force in the static analysis. Finally general static structural analysis can be executed to obtain resultant stress state of the tunnel at the time $t_{m}$ and it can be considered as the approximate maximum stress state.

\subsection{Definition of Damage State}

Seismic fragility curves are required to be developed according to the variation of damage index with increasing of seismic intensity for different levels of damage states. Due to the lack of information available on damage indexes and related parameters for the development of tunnel fragility curves, the damage index (DI) is assumed to be the ratio between the actual bending moment $(M)$ and capacity bending moment $\left(M_{R d}\right)$ of the tunnel cross section. A definition based on moments is compatible with the use of displacements, according to the equal displacement approximation. It is assumed that the behavior of tunnel is approximated to that of an elastic beam subjected to deformations imposed by the surrounding soil due to seismic waves propagating perpendicular to the tunnel axis. With help of the previous experience on damages of tunnels and engineering judgment, a set of 4 different damage states is introduced in this study as shown in Table $\mathbf{1}$.

\subsection{Fragility Curve Development}

It is generally assumed that the fragility curves can be expressed in the form of two-parameters of distribution functions as shown below [2].

$$
P(I M=x)=\Phi\left(\frac{\ln x-\mu}{\beta}\right) .
$$

where $P(I M=x)$ represents the probability of collapse, given a ground motion with $I M=x, \Phi()$ is the normal cumulative distribution function, $\mu$ and $\beta$ are the mean and standard deviation of $\ln I M$, respectively.

The Equation (1) can be expressed in terms of the median parameter as.

$$
P(I M=x)=\Phi\left(\frac{\ln (x / \theta)}{\beta}\right) .
$$

where $\theta$ represents the median of the fragility function (the IM level with $50 \%$ probability of collapse)

The estimation of these two-parameters is done by the maximum likelihood estimates (MLE) treating each IM level of tunnel damage as a realization from a Bernoulli experiment. The likelihood is expressed as [4]:

$$
\text { Likelihood }=\prod_{j=1}^{m}\left(\begin{array}{c}
n_{j} \\
z_{j}
\end{array}\right) p_{j}^{z_{j}}\left(1-p_{j}^{z_{j}}\right)^{n_{j}-z_{j}} .
$$

where $p_{j}$ represents the probability that a ground motion with $I M=x_{j}$ will cause collapse, $z_{j}$ is the num-

Table 1. Damage state provided by Pitilakis [5].

\begin{tabular}{cccc}
\hline None & Minor & Moderate & Extensive \\
\hline $\mathrm{DI}<=1.0$ & $1.0<\mathrm{DI}<=1.5$ & $1.5<\mathrm{DI}<=2.5$ & $2.5<\mathrm{DI}<=3.5$ \\
\hline
\end{tabular}


ber of collapses out of $n_{j}$ ground motions, $m$ represents the number of IM level.

The final goal is to choose the function that produces the highest probability of observing collapses. Therefore, estimates of fragility function parameters can be obtained by maximizing the following likelihood function.

$$
\{\hat{\mu}, \hat{\theta}\}=\max \prod_{i=1}^{m}\left(\begin{array}{l}
n_{j} \\
z_{j}
\end{array}\right) \Phi\left(\frac{\ln \left(x_{j} / \theta\right)}{\beta}\right)^{z_{j}}\left(1-\Phi\left(\frac{\ln \left(x_{j} / \theta\right)}{\beta}\right)\right)^{n_{j}-z_{j}} .
$$

The parameters which maximized likelihood function (4) will also maximize the log likelihood function below:

$$
\{\hat{\mu}, \hat{\theta}\}=\max \sum_{i=1}^{m}\left\{\ln \left(\begin{array}{c}
n_{j} \\
z_{j}
\end{array}\right)+z_{j} \ln \Phi\left(\frac{\ln \left(x_{j} / \theta\right)}{\beta}\right)+\left(n_{j}-z_{j}\right) \ln \left(1-\Phi\left(\frac{\ln \left(x_{j} / \theta\right)}{\beta}\right)\right)\right\}
$$

\section{Numerical Example}

An actual one-story two-cell reinforced concrete tunnel (cut and cover section) is considered in this numerical example. As shown in Figure 1, the width of the tunnel is $29.3 \mathrm{~m}$ and the height is $7.8 \mathrm{~m}$. The structure consists of two reinforced concrete deck slabs (the thickness of the top and bottom slab are $1.2 \mathrm{~m}$ and $1.3 \mathrm{~m}$, respectively) and it is supported by $1.0 \mathrm{~m}$ thickness walls in both sides and a column in the middle $(0.6 \mathrm{~m} \times 0.74 \mathrm{~m})$. The soil profile around the tunnel consists of three layers namely sand, weathered soil, and rock, respectively. The properties of concrete tunnel and soil layers in this example are given in Table 2 and Table $\mathbf{3}$ in details.

In order to established the fragility curves for different damage states defined above, 200 artificial time histories (20 different time histories for each seismic level) based on the design spectrum [6] are randomly utilized as input data for the application of free-field soil analysis approach. The acceleration profiles at $t_{m}$ when displacement value between the top and bottom of the tunnel is largest are obtained at each layers of ground system. The process of RAM is illustrated in Figure 2.

The tunnel is analyzed with the MIDAS Civil finite element program [3], using the two-dimensional model. According to this model, the structure is composed of vertical column and horizontal beam with specified sections in Table 4. Body forces converted from response acceleration results at each ground layers are applied
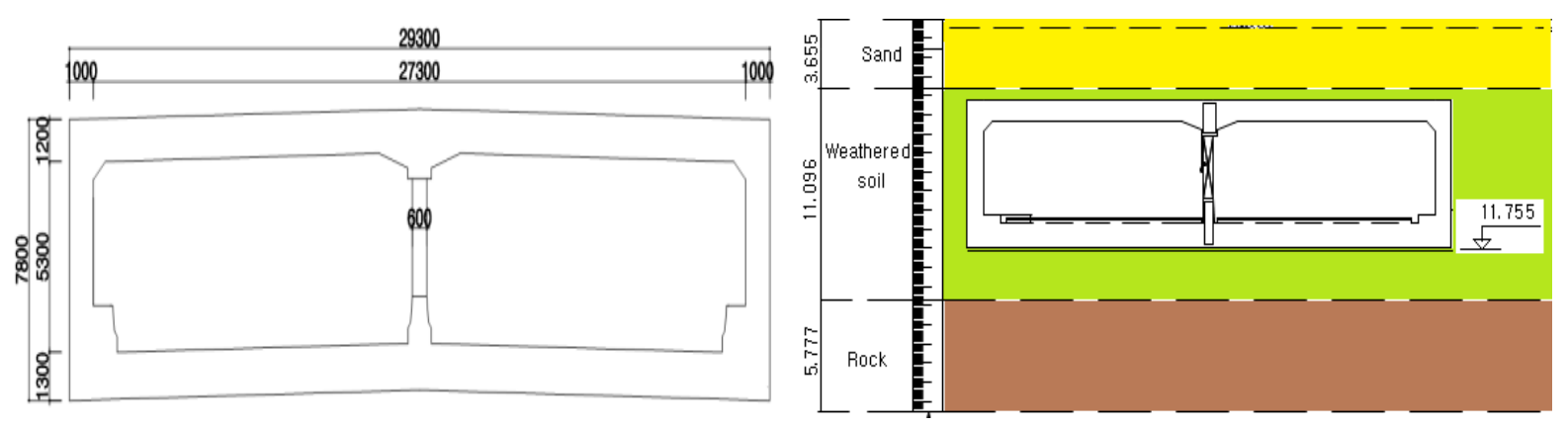

Figure 1. Dimension and ground condition of the example.

Table 2. Properties of reinforced concrete structure.

\begin{tabular}{cccccc}
\hline Material & Poisson Ratio & $v$ & Elastic Modulus $E_{c}(\mathrm{kPa})$ & Unit mass $\rho\left(\mathrm{t} / \mathrm{m}^{3}\right)$ & Damping \\
\hline Concrete & 0.17 & 28693 & 2.5 & 0.5 \\
\hline
\end{tabular}

Table 3. Properties of soils.

\begin{tabular}{cccc}
\hline Soil name & Shear wave velocity $(\mathrm{m} / \mathrm{s})$ & Poisson Ratio & Unit weight $\left(\mathrm{t} / \mathrm{m}^{3}\right)$ \\
\hline Sand & 275 & 0.35 & 1.8 \\
Weathered soil & 500 & 0.35 & 2.0 \\
Rock & 1500 & 0.25 & 2.3 \\
\hline
\end{tabular}




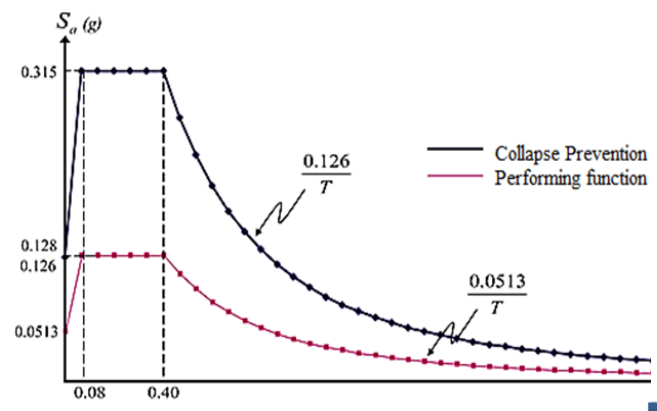

Design spectrum
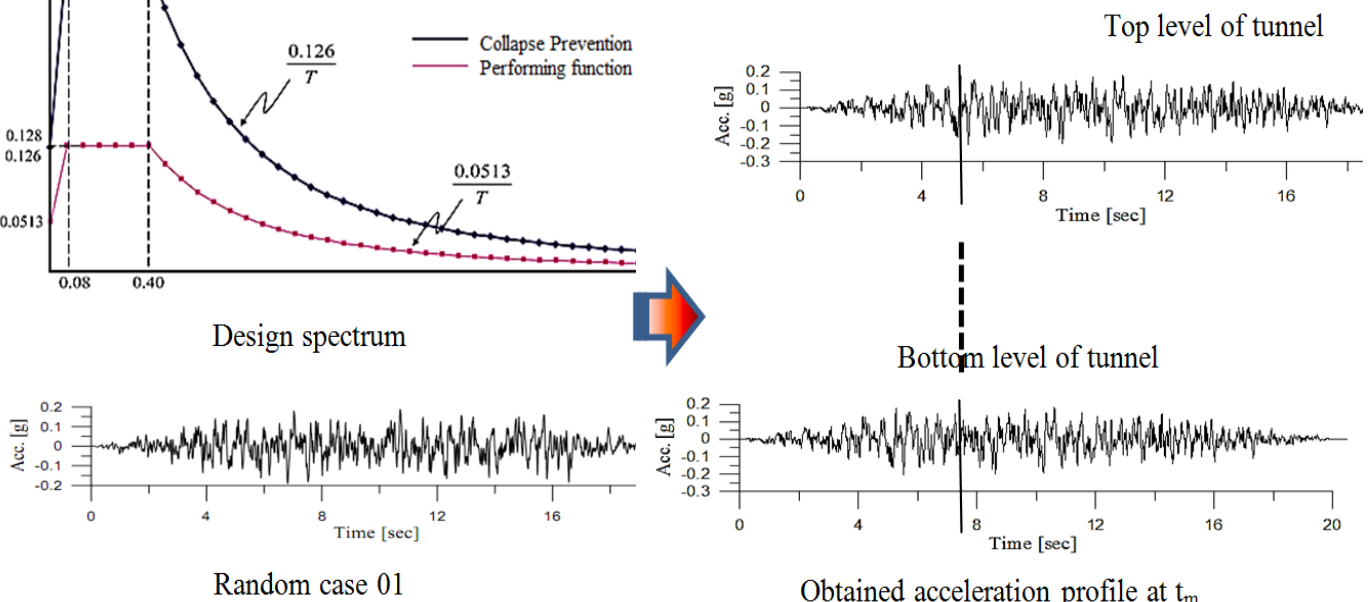

Obtained acceleration profile at $t_{m}$

Figure 2. Response acceleration method process.

Table 4. Specification section of the structure model.

\begin{tabular}{cccc}
\hline Section name & Longitudinal $(\mathrm{m}) b$ & Thickness $(\mathrm{m}) h$ & Area $\left(\mathrm{m}^{2}\right) A=b h$ \\
\hline Upper slab & 2.0 & 1.2 & 2.4 \\
Lower slab & 2.0 & 1.3 & 2.6 \\
Walls & 2.0 & 1.0 & 2.0 \\
Column (CTC $=4 \mathrm{~m})$ & 0.6 & 0.74 & 0.44 \\
\hline
\end{tabular}

to the tunnel. Static analysis is performed and bending moment results of the structure which illustrated in Figure $\mathbf{3}$ are obtained.

From the results of structure analysis, moment data at weakest point (the intersection point between vertical column and horizontal beam) are collected. Then, the failure probabilities for three damage states according to Table 1 are evaluated at each increment of seismic intensity in the Table 5 where (1), (2), and (3) are minor damage, moderate damage and extensive damage, respectively and $I M$ is the intensity measure of the ground acceleration in terms of standard gravity.

Two statistical parameters for the fragility curve of the tunnel are obtained using proposed method and fragility curves are derived from it. The Maximum Likelihood method applying to obtain two-parameters is clarified in Figure 4. The median peak ground acceleration at each damage state is acquired with its corresponding standard deviation value summarized in Table 6.

Figure 5 displays the computed fragility curves which associated with damage state definition provided in Table 1. This computation can be performed using EXCEL program in the study.

The figure shows that the fragility curves for various damage indexes which provided in Table $\mathbf{1}$ have similar shape. The number of failures dramatically increases with the slightly rise of seismic intensity which is satisfactory agreement compared to empirical fragility curves provided by [5]. The definition of damage states for tunnel structure needs to be investigated further since there is no universal guideline for it in the profession.

\section{Conclusion}

A simple yet comprehensive numerical methodology is proposed for fragility assessment of an underground tunnel subjected to seismic loading by integrating responses acceleration method (RAM) and Maximum Likelihood approach. It is elaborated with an illustrative numerical example and found to be computationally efficient. However, there is a necessity for further study on various types of structures and adequate-rational damage states need to be established in the development of seismic fragility curves for tunnel structure. 

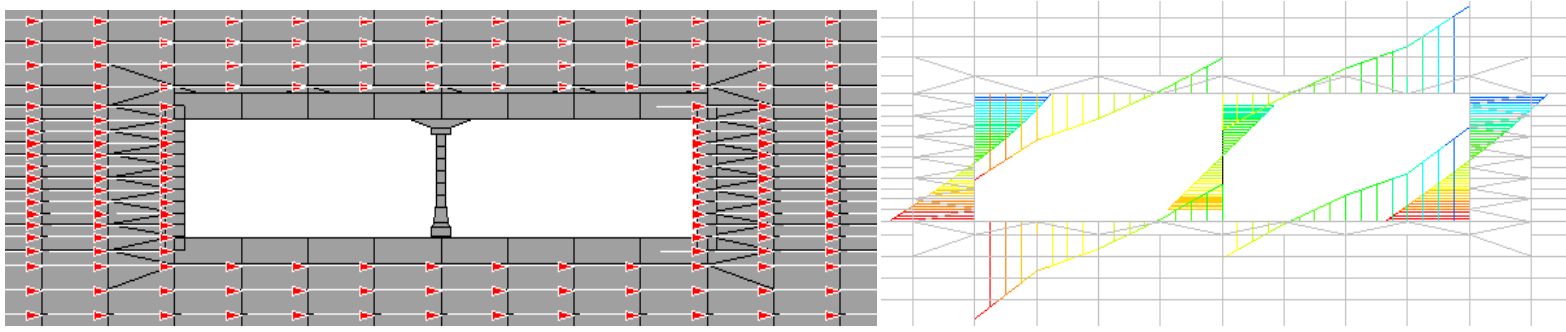

Figure 3. Model example and bending moment result.

\begin{tabular}{|c|c|c|c|c|c|c|}
\hline IM & $\begin{array}{c}\text { Number of } \\
\text { analyses }\end{array}$ & $\begin{array}{c}\text { Number of } \\
\text { collapses }\end{array}$ & $\begin{array}{c}\text { Fraction } \\
\text { causing collapse }\end{array}$ & $\begin{array}{c}\text { Theoretical fragility } \\
\text { function }\end{array}$ & Likelihood & Log likelihood \\
\hline 0.05 & 20 & 0 & 0.00 & 0.00 & 1.000 & 0.000 \\
\hline 0.065 & 20 & 0 & 0.00 & 0.00 & 0.913 & -0.091 \\
\hline 0.085 & 20 & 7 & 0.35 & 0.31 & 0.173 & -1.753 \\
\hline 0.1 & 20 & 15 & 0.75 & 0.79 & 0.186 & -1.679 \\
\hline 0.125 & 20 & 20 & 1.00 & 0.99 & 0.901 & -0.104 \\
\hline 0.135 & 20 & 20 & 1.00 & 1.00 & 0.985 & -0.015 \\
\hline 0.15 & 20 & 20 & 1.00 & 1.00 & 0.999 & -0.001 \\
\hline 0.2 & 20 & 20 & 1.00 & 1.00 & 1.000 & 0.000 \\
\hline 0.22 & 20 & 20 & 1.00 & 1.00 & 1.000 & 0.000 \\
\hline 0.24 & 20 & 20 & 1.00 & 1.00 & 1.000 & 0.000 \\
\hline 0.25 & 20 & 20 & 1.00 & & & \\
& & & & & & \\
\end{tabular}

Figure 4. Two parameters obtained using Likelihood approach.

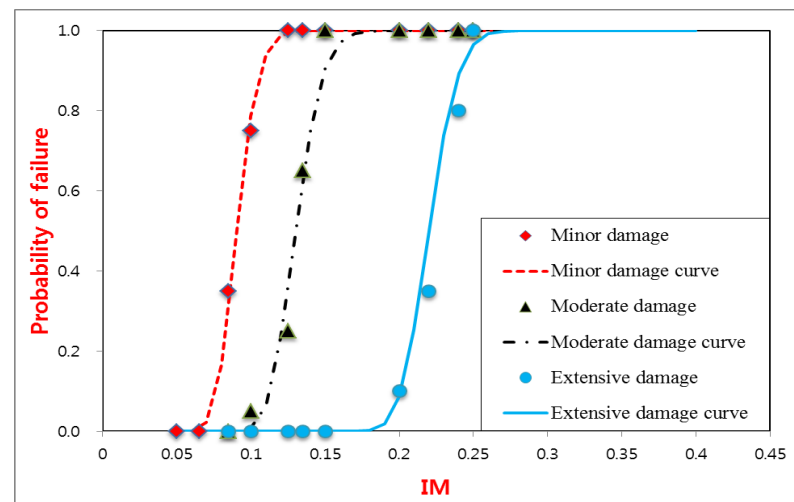

Figure 5. Fragility analysis results.

Table 5. Obtained failure probability data.

\begin{tabular}{ccccccc}
\hline \multirow{2}{*}{$I M(\mathrm{~g})$} & \multicolumn{3}{c}{ Number of failure } & \multicolumn{3}{c}{ Probability of failure } \\
\cline { 2 - 7 } & $(1)$ & $(2)$ & $(3)$ & $(1)$ & $(2)$ & $(3)$ \\
\hline 0.060 & 0 & 0 & 0 & 0.00 & 0.00 & 0.00 \\
0.085 & 7 & 0 & 0 & 0.35 & 0.05 & 0.00 \\
0.100 & 15 & 1 & 0 & 0.75 & 0.25 & 0.00 \\
0.125 & 20 & 5 & 0 & 1.00 & 0.65 & 0.00 \\
0.135 & 20 & 13 & 0 & 1.00 & 1.00 & 0.00 \\
0.150 & 20 & 20 & 0 & 1.00 & 1.00 & 0.00 \\
0.200 & 20 & 20 & 7 & 1.00 & 1.00 & 0.10 \\
0.220 & 20 & 20 & 16 & 1.00 & 1.00 & 0.35 \\
0.240 & 20 & 20 & 20 & 1.00 & 1.00 & 0.80 \\
0.250 & 20 & 20 & & 1.00 & 1.00 & 1.00 \\
\hline
\end{tabular}


Table 6. Summary static parameter value.

\begin{tabular}{|c|c|c|c|}
\hline Damage state & Minor & Moderate & Extensive \\
\hline Median $\theta$ & 0.09 & 0.13 & 0.22 \\
\hline Standard Deviation $\beta$ & 0.13 & 0.11 & 0.07 \\
\hline
\end{tabular}

\section{Acknowledgements}

This research was supported by Basic Science Research Program through the National Research Foundation of Korea (NRF) funded by the Ministry of Education, Science and Technology (2012R1A1A4A01015343).

\section{References}

[1] Katayama, I. (1990) Study on Fundamental Problems in Seismic Design Analyses of Critical Structures and Facilities.

[2] Shinouka, M., Feng, M.Q., Kim, H., Uzawa, T. and Ueda, T. (2001) Statistical Analysis of Fragility Curves.

[3] Midas-IT (2011) MIDAS Civil On-Line Manual. http://manual.midasuser.com/EN_TW/civil/791/index.htm

[4] Baker, J.W. (2011) Fitting Fragility Functions to Structural Analysis Data Using Maximum Likelihood Estimation. http://web.stanford.edu/ bakerjw/fragility/archived_versions/Baker_(2011)_fragility_fitting.pdf

[5] Pitilakis, K. (1995) Fragility Function for Roadway System Elements. SYNER-G, No. 244061.

[6] KBC (2013) Korea Building Code. 